\title{
Bleeding complications in critically ill patients with liver cirrhosis
}

\author{
Jaeyoung Cho ${ }^{1}$, Sun Mi Choi ${ }^{1}$, Su Jong Yu², Young Sik Park ${ }^{1}$, Chang-Hoon Lee ${ }^{1}$, Sang-Min Lee ${ }^{1}$, Jae-Joon Yim,
} Chul-Gyu Yoo', Young Whan Kim, Sung Koo Han ${ }^{1}$, and Jinwoo Lee ${ }^{1}$

${ }^{1}$ Division of Pulmonary and Critical Care Medicine, Department of Internal Medicine, Seoul National University Hospital, Seoul; ${ }^{2}$ Department of Internal Medicine and Liver Research Institute, Seoul National University College of Medicine, Seoul, Korea

\author{
Received: May 23, 2014 \\ Revised : November 10, 2014 \\ Accepted: December 17, 2014

\section{Correspondence to} \\ Jinwoo Lee, M.D. \\ Division of Pulmonary and \\ Critical Care Medicine, \\ Department of Internal Medicine, \\ Seoul National University Hos- \\ pital, 101 Daehak-ro, Jongno-gu, \\ Seoul 03080, Korea \\ Tel: +82-2-2072-7593 \\ Fax: +82-2-762-9662 \\ E-mail: realrain7@gmail.com
}

Background/Aims: Patients with liver cirrhosis (LC) are at risk for critical events leading to Intensive Care Unit (ICU) admission. Coagulopathy in cirrhotic patients is complex and can lead to bleeding as well as thrombosis. The aim of this study was to investigate bleeding complications in critically ill patients with LC admitted to a medical ICU (MICU).

Methods: All adult patients admitted to our MICU with a diagnosis of LC from January 2006 to December 2012 were retrospectively assessed. Patients with major bleeding at the time of MICU admission were excluded from the analysis.

Results: A total of 205 patients were included in the analysis. The median patient age was 62 years, and $69.3 \%$ of the patients were male. The most common reason for MICU admission was acute respiratory failure (45.4\%), followed by sepsis (27.3\%). Major bleeding occurred in 25 patients (12.2\%). The gastrointestinal tract was the most common site of bleeding (64\%), followed by the respiratory tract (20\%). In a multivariate analysis, a low platelet count at MICU admission (odds ratio [OR], 0.98; 95\% confidence interval [CI], 0.97 to 0.99 ) and sepsis (OR, 8.35; $95 \%$ CI, 1.04 to 67.05 ) were independent risk factors for major bleeding. The ICU fatality rate was significantly greater among patients with major bleeding $(84.0 \%$ vs. $58.9 \%$, respectively; $p=0.015$ ).

Conclusions: Major bleeding occurred in $12.2 \%$ of critically ill cirrhotic patients admitted to the MICU. A low platelet count at MICU admission and sepsis were associated with an increased risk of major bleeding during the MICU stay. Further study is needed to better understand hemostasis in critically ill patients with LC.

Keywords: Liver cirrhosis; Intensive care unit; Hemorrhage; Risk factors

\section{INTRODUCTION}

Liver cirrhosis (LC) is a major health problem worldwide, and cirrhotic patients are subject to critical events leading to Intensive Care Unit (ICU) admission. While previously believed to be naturally "auto-anticoagulated," patients with LC experience a unique coagulopathy in which they are not only prone to hemorrhage but also remain susceptible to thrombosis [1-4]. The coagulation system in cirrhotic patients is rebalanced, owing to a parallel reduction in procoagulant and anticoagulant factors. Despite this rebalancing, the coagulation system in patients with LC is fragile, and may cause hemorrhage or thrombosis, depending on the prevailing risk factors [5].

Recent literature supports the use of pharmacologic 
venous thromboembolism (VTE) prophylaxis in critically ill patients with LC [6-8]. However, the rate of thromboprophylaxis in this population has been reported to be suboptimal $[2,9]$. Since prophylaxis for VTE carries an increased risk of bleeding and other complications in patients with cirrhosis, it would be helpful to be able to identify those patients who are at risk for bleeding or for thrombosis. However, there is currently no evidence to support the use of routine coagulation tests to predict bleeding or thrombotic risk in critically ill cirrhotic patients [10]. Moreover, bleeding complications in cirrhotic patients in the Medical ICU (MICU) have not been well described.

The aim of this study was to investigate bleeding complications in critically ill patients with LC admitted to the MICU, and to identify risk factors for major bleeding.

\section{METHODS}

\section{Study population}

A retrospective chart review was conducted of patients with LC who were admitted to the MICU at Seoul National University Hospital between January 2006 and December 2012. The patients were adults, aged 18 years or older, and LC was defined based on clinical or histologic criteria. Patients with major bleeding at the time of MICU admission were excluded. During the study period, 273 cirrhotic patients were admitted to the MICU; 68 were excluded for major bleeding at the time of admission. This study was approved by the Institutional Review Board of Seoul National University Hospital.

\section{Data collection}

The following data were collected: demographic characteristics; reason for MICU admission; cause of cirrhosis; MICU admission creatinine level, international normalized ratio (INR), bilirubin level, albumin level, hematocrit, and platelet count; need for vasopressors or inotropics, mechanical ventilation, or renal replacement therapy; history of malignancy; sepsis; previous liver transplantation; comorbidities; Child-Pugh score; model of end-stage liver disease; acute physiology and chronic health evaluation II; sequential organ failure assessment; bleeding events and sites; and use of pharma- cologic VTE prophylaxis.

Bleeding was defined as major if, in the absence of another cause, it fulfilled any of the five following criteria: (1) life-threatening bleeding with hypovolemic shock; (2) life-threatening bleeding into a critical site (e.g., intracranial or pericardial); (3) other critical bleeding (e.g., epidural, intraocular, or intra-articular); (4) bleeding requiring an invasive procedure (e.g., surgery or embolization); or (5) clinically important bleeding requiring the transfusion of two or more units of packed red blood cells, or associated with a decrease in systolic blood pressure of at least $20 \mathrm{mmHg}$ or an increase in heart rate of at least 20 beats per minute. Bleeding that did not satisfy any of these criteria was defined as minor bleeding $[11,12]$.

The development of symptomatic deep vein thrombosis (DVT) was confirmed by venous Doppler ultrasound of the lower limbs, and the development of symptomatic pulmonary embolism was confirmed by spiral computed tomography (CT) of the chest or by a high-probability ventilation-perfusion scan. These tests were ordered by the treating physicians based on clinical suspicion.

\section{Statistical analysis}

All data are shown as medians and interquartile ranges, or as percentages, as appropriate. Univariate and multivariate analyses were performed; the results are described with odds ratios (ORs) and 95\% confidence intervals (CIs). p values less than 0.05 were considered to have statistical significance. All statistical analyses were performed using SPSS version 19.0 (IBM Co., Armonk, NY, USA).

\section{RESULTS}

\section{Patient characteristics}

A total of 205 patients were included in the final analysis. The median age of the patients was 62 years (interquartile range, 53 to 70 ), and $69.3 \%$ of the patients were male (Table 1). There were no differences between those with major bleeding and those without in age, sex, body mass index, reason for MICU admission, or etiology of LC. The most common reason for MICU admission was acute respiratory failure (93/205, 45.4\%), followed by sepsis $(56 / 205,27.3 \%)$. Hepatitis B was the most common 
Table 1. Baseline characteristics

\begin{tabular}{|c|c|c|c|c|}
\hline Characteristic & Total $(\mathrm{n}=205)$ & $\begin{array}{l}\text { Without major bleeding } \\
\qquad(\mathrm{n}=18 \mathrm{o})\end{array}$ & $\begin{array}{l}\text { With major bleeding } \\
(\mathrm{n}=25)\end{array}$ & $p$ value \\
\hline \multicolumn{5}{|l|}{ Patient characteristic } \\
\hline Age, yr & $62(53-70)$ & $62(53 \cdot 3-70)$ & $61(49.5-68.5)$ & 0.825 \\
\hline Male sex & $142(69 \cdot 3)$ & $126(70)$ & $16(64.0)$ & 0.542 \\
\hline Body mass index, $\mathrm{kg} / \mathrm{m}^{2}$ & $22.6\left(20.5^{-25.2)}\right.$ & $22.7(20.5-25.4)$ & $22.6(20.7-24.4)$ & 0.377 \\
\hline \multicolumn{5}{|l|}{ Reason for MICU admission } \\
\hline Sepsis & $56(27 \cdot 3)$ & $48(26.7)$ & $8(32)$ & 0.748 \\
\hline Respiratory distress & $93(45 \cdot 4)$ & $82(45.6)$ & $11(44)$ & 1.000 \\
\hline Hepatic encephalopathy & $2(1.0)$ & $2(1.1)$ & 0 & 1.000 \\
\hline Cardiac arrhythmia & $7(3.4)$ & $6(3 \cdot 3)$ & $1(4)$ & 1.000 \\
\hline Postoperative care & $23(11.2)$ & $22(12.2)$ & $1(4)$ & \\
\hline Continuous renal replacement therapy & $23(11.2)$ & $19(10.6)$ & $4(16)$ & 0.378 \\
\hline Anaphylactic shock & $1(0.5)$ & $1(0.6)$ & o & 1.000 \\
\hline \multicolumn{5}{|l|}{ Etiology of liver cirrhosis } \\
\hline Hepatitis B & $112(54.6)$ & $100(55 \cdot 6)$ & $12(48)$ & 0.619 \\
\hline Hepatitis C & $29(14.1)$ & $25(13 \cdot 9)$ & $4(16)$ & 1.000 \\
\hline Alcoholic & $33(16.1)$ & $29(16.1)$ & $4(16)$ & 0.835 \\
\hline Autoimmune & $6(2.9)$ & $5(2.8)$ & $1(4)$ & 1.000 \\
\hline Cryptogenic & $18(8.8)$ & $14(7.8)$ & $4(16)$ & 0.325 \\
\hline Other $^{\mathrm{a}}$ & $7(3 \cdot 4)$ & $7(3 \cdot 9)$ & 0 & 0.677 \\
\hline
\end{tabular}

Values are presented as median (interquartile range) or number (\%).

MICU, Medical Intensive Care Unit.

${ }^{a}$ Among the seven patients, five were diagnosed with cardiac cirrhosis, one with Budd-Chiari syndrome, and one with biliary cirrhosis.

etiologic agent, accounting for the majority of LC cases (112/205, 54.6\%), followed by alcohol and hepatitis C, accounting for 33 cases (16.1\%) and 29 cases (14.1\%), respectively.

\section{Incidence of and risk factors for bleeding}

Major or minor bleeding occurred in 42 patients (20.5\%), and major bleeding occurred in 25 patients (12.2\%). One patient had two major bleeding events (respiratory tract and genitourinary tract). The gastrointestinal tract was the most common site of major bleeding $(16 / 25,64.0 \%)$, followed by the respiratory tract $(5 / 25,20.0 \%$ ) (Table 2 ).

Of the 16 major gastrointestinal bleeds, 15 were upper gastrointestinal bleeds and one was a lower gastrointestinal bleed confirmed by colonoscopy. Of the 15 upper gastrointestinal bleeds, seven were confirmed by esophagogastroduodenoscopy. The remaining eight patients were diagnosed by clinical suspicion and bloody Levin tube drainage. Portal hypertensive gastropathy was the most common type of bleeding (three patients) among those who underwent esophagogastroduodenoscopy. Other major gastrointestinal bleeding sources were esophageal varix (one case), peptic ulcer (two cases), and angiodysplasia (one case). The gastrointestinal tract was also the most common site of minor bleeding ( $7 / 17$, 41.2\%), and all minor gastrointestinal bleeds were from the upper gastrointestinal tract.

Five patients $(5 / 25,20.0 \%)$ had major respiratory tract bleeding. Two bled from tracheostomy sites, two into endotracheal tube aspirates, and one into chest tube drainage. Of these five patients, one underwent a bronchoscopy, which identified a bronchogenic lung cancer as the source of the bleeding. All four patients with minor respiratory tract bleeding had bloody endotracheal tube aspirates, none of which led to bronchoscopic evaluation. 
Table 2. Bleeding sites for major or minor bleeding among cirrhotic patients

\begin{tabular}{lcc}
\hline Bleeding site & Major bleeding $(\mathrm{n}=25)$ & Minor bleeding $(\mathrm{n}=17)$ \\
\hline Gastrointestinal & $16(64.0)$ & $7(41.2)$ \\
Respiratory & $5(20.0)$ & $4(23.5)$ \\
Genitourinary & $2(8.0)$ & $4(23.5)$ \\
Intracranial & $3(12.0)$ & 0 \\
Vascular catheter or tube insertion site & 0 & $1(5.9)$ \\
Skin bleeding & 0 & $1(5.9)$ \\
\hline
\end{tabular}

Values are presented as number (\%). One patient had two major bleeding events (respiratory tract bleeding and genitourinary bleeding).

Three patients $(3 / 25,12.0 \%)$ with bilateral pupil dilatation and an absence of light reflexes were diagnosed with intracranial bleeding by brain CT. All of these patients had intracerebral hemorrhages. After discussions between the physicians and families, surgical intervention was declined for these patients on the basis of poor predicted neurological outcomes.

To identify the risk factors associated with the development of major bleeding, univariate and multivariate analyses were performed. In a univariate analysis for major bleeding, platelet count on the day of MICU admission ( $p=0.028)$, sepsis $(p=0.036)$, and diabetes mellitus $(p=0.041)$ were significantly associated with major bleeding (Table 3). By multivariate analysis, however, a low platelet count on the day of MICU admission (OR, $0.987 ; 95 \% \mathrm{CI}, 0.976$ to $0.998 ; p=0.025)$ and sepsis (OR, 8.352; $95 \%$ CI, 1.040 to 67.056 ; $p=0.046$ ) were independent risk factors for major bleeding. The platelet count on admission was significantly lower in the group with major bleeding (median, $41.0 \times 10^{9} / \mathrm{L}$ vs. $76.5 \times 10^{9} / \mathrm{L}$, respectively; $p=0.025)$. Sepsis was also more prevalent in the group with major bleeding than in the group without $(96.0 \%$ vs. $73.3 \%$, respectively; $p=0.046)$.

\section{Fatality analysis}

The duration of mechanical ventilation and the length of hospital stay were not significantly different between the groups with and without major bleeding (Table 4). ICU stay length was significantly longer in patients with major bleeding (median, 12 days vs. 6 days, respectively; $p=0.002$ ). ICU fatalities were significantly higher in patients with major bleeding (84.0\% vs. $58.9 \%$, respectively; $p=0.015$ ), as were in-hospital fatalities (96.0\% vs. $67.8 \%$, respectively; $p=0.003$ ). The bleeding-attributable
ICU fatality rate was $44 \%$ (11/25). Of the eleven bleeding-attributable deaths, gastrointestinal bleeding was the most common cause (six patients), followed by intracranial bleeding (three patients) and respiratory tract bleeding (two patients). All 11 patients died within 2 days of major bleeding; three of them died on the day of bleeding. All-cause fatalities and length of hospital stay did not differ between the group with no bleeding and that with minor bleeding. ICU fatalities differed significantly between the group with major bleeding and that with no bleeding ( $84.0 \%$ vs. $58.3 \%$, respectively; $p=0.02$ ).

\section{Practice of venous thromboembolism prophylaxis}

Evaluations for VTE were initiated only when clinically suspected. Six patients $(6 / 205,2.9 \%)$ underwent screening tests (four by pulmonary CT angiography and two by venous Doppler ultrasound of the lower extremities). Among them, one patient was newly diagnosed with DVT and had not received pharmacologic thromboprophylaxis. During their stays in the MICU, only eight patients $(8 / 205,3.9 \%)$ received pharmacologic prophylaxis for VTE. Others received anticoagulation therapy for mechanical heart valves (four patients), atrial fibrillation (one patient), acute coronary syndrome (three patients), and VTE treatment (one patient). Of the 17 patients who were given heparin or warfarin, only one patient had a major bleeding episode, from a tracheostomy site, which resolved with conservative management after 2 days.

\section{DISCUSSION}

Among 205 critically ill patients with LC, clinically significant major bleeding occurred in 25 (12.2\%). The most 
Table 3. Risk factors for major bleeding

\begin{tabular}{|c|c|c|c|c|c|}
\hline \multirow{2}{*}{ Patient factor } & \multirow{2}{*}{$\begin{array}{l}\text { Without major bleeding } \\
\qquad(\mathrm{n}=180)\end{array}$} & \multirow{2}{*}{$\begin{array}{l}\text { With major bleeding } \\
\qquad(\mathrm{n}=25)\end{array}$} & \multirow{2}{*}{$\begin{array}{l}\text { Univariate analysis } \\
\qquad p \text { value }\end{array}$} & \multicolumn{2}{|c|}{ Multivariate analysis } \\
\hline & & & & OR $(95 \% \mathrm{CI})$ & $p$ value \\
\hline \multicolumn{6}{|l|}{ Patient characteristic } \\
\hline Age, yr & & & 0.825 & & \\
\hline$<60$ & $75(41.7)$ & $11(44.0)$ & & & \\
\hline$\geq 60$ & $105(58.3)$ & $14(56.0)$ & & & \\
\hline Male sex & $126(70.0)$ & $16(64.0)$ & 0.542 & & \\
\hline \multicolumn{6}{|l|}{ Medical condition } \\
\hline History of malignancy & $116(64.4)$ & $19(76)$ & 0.254 & & \\
\hline Liver transplantation & $10(5 \cdot 6)$ & $3(12.0)$ & 0.200 & & \\
\hline Coronary artery disease & $10(5 \cdot 6)$ & $2(8.0)$ & 0.644 & & \\
\hline COPD & $9(5.0)$ & 0 & 0.604 & & \\
\hline Diabetes mellitus & $66(36.7)$ & $4(16.0)$ & 0.041 & $0.37(0.11-1.22)$ & 0.105 \\
\hline Hypertension & $44(24 \cdot 4)$ & $5(20)$ & 0.625 & & \\
\hline Heart failure & $15(8.3)$ & $1(4.0)$ & 0.699 & & \\
\hline End-stage renal disease & $84(46.7)$ & $16(64)$ & 0.104 & & \\
\hline Mechanical ventilation & $156(86.7)$ & $23(92.0)$ & 0.748 & & \\
\hline Use of vasoactive agents & $142(78.9)$ & $23(92.0)$ & 0.177 & & \\
\hline Sepsis & $132(73 \cdot 3)$ & $24(96.0)$ & 0.036 & $8.35(1.04-67.05)$ & 0.046 \\
\hline Hematocrit, \% & $26.9(22.6-30.8)$ & $25.7(21.6-27.7)$ & 0.066 & & \\
\hline Creatinine, mg/dL & $2.0(1.1-3.4)$ & $2.3(1.2-4.0)$ & 0.972 & & \\
\hline$>1.5$ & $109(60.6)$ & $15(60)$ & 0.958 & & \\
\hline INR & $1.9(1.4-2.7)$ & $2.1(1.6-2.6)$ & 0.854 & & \\
\hline$>1.5$ & $128(71.1)$ & $21(84)$ & 0.184 & & \\
\hline APTT, sec & $52.2(41.1-66.3)$ & $55.3(43.5-64.9)$ & 0.996 & & \\
\hline Bilirubin, mg/dL & $4.8(2.0-13.8)$ & $6.4(3.5-19.8)$ & 0.288 & & \\
\hline Albumin, g/dL & $2.5(2.1-2.9)$ & $2.5(2.3-3.0)$ & 0.701 & & \\
\hline Platelets, $10^{9} / \mathrm{L}$ & $67.5(36.0-116.8)$ & $41.0(22.5-84.0)$ & 0.028 & $0.98(0.97-0.99)$ & 0.025 \\
\hline Child-Pugh score & $11(9-13)$ & $13(11-14)$ & 0.173 & & \\
\hline A & $7(3 \cdot 9)$ & 0 & 0.428 & & \\
\hline $\mathrm{B}$ & $48(26.7)$ & $5(20)$ & & & \\
\hline C & $125(69.4)$ & $20(80)$ & & & \\
\hline MELD score & $27(18-35)$ & $28(24-36.5)$ & 0.382 & & \\
\hline APACHE II score & $30(22-35.8)$ & $25(19 \cdot 5-32.5)$ & 0.087 & & \\
\hline SOFA score & $14(11-17)$ & $14(10.5-16.5)$ & 0.976 & & \\
\hline \multicolumn{6}{|l|}{ Medications used } \\
\hline Anti-platelet agent & $14(7.8)$ & $2(8.0)$ & 1.000 & & \\
\hline LMWH or UFH & $13(7 \cdot 2)$ & $1(4 \cdot 0)$ & 1.000 & & \\
\hline Warfarin & $6(3 \cdot 3)$ & 0 & 1.000 & & \\
\hline
\end{tabular}

Values are presented as number (\%) or median (interquartile range).

OR, odds ratio; CI, confidence interval; COPD, chronic obstructive pulmonary disease; INR, international normalized ratio; APTT, activated partial thromboplastin time; MELD, model of end-stage liver disease; APACHE II, acute physiology and chronic health evaluation II; SOFA, sequential organ failure assessment; LMWH, low molecular weight heparin; UFH, unfractionated heparin. 
Table 4. Clinical outcomes, with and without major bleeding

\begin{tabular}{lcccc}
\hline \multicolumn{1}{c}{ Variable } & $\begin{array}{c}\text { Without major bleeding } \\
(\mathrm{n}=180)\end{array}$ & $\begin{array}{c}\text { With major bleeding } \\
(\mathrm{n}=25)\end{array}$ & p value & OR (95\% CI $)$ \\
\hline Length of MV, day & $6(3-13.8)$ & $13(8-20)$ & 0.931 & $1.00(0.99-1.00)$ \\
Hospital LOS, day & $29(15-46.8)$ & $19(14-39)$ & 0.358 & $0.99(0.97-1.00)$ \\
ICU LOS, day & $6(3-12)$ & $12(8-17.5)$ & 0.002 & $1.06(1.02-1.10)$ \\
Hospital fatality & $122(67.8)$ & $24(96.0)$ & 0.003 & $11.41(1.50-86.41)$ \\
ICU fatality & $106(58.9)$ & $21(84.0)$ & 0.015 & $3.66(1.20-11.11)$ \\
Bleeding-attributable ICU fatality & 0 & $11(44.0)$ & - & - \\
\hline
\end{tabular}

Values are presented as median (interquartile range) or number (\%).

OR, odds ratio; CI, confidence interval; MV, mechanical ventilation; LOS, length of stay; ICU, Intensive Care Unit.

common site of bleeding was gastrointestinal (16/25, 64.0\%). Most previous studies of patients with LC have focused on gastrointestinal bleeding, especially variceal bleeding; studies of other bleeding complications are rare [13-15].

Portal hypertension, endothelial dysfunction, bacterial infection, and renal failure are important factors contributing to the bleeding diathesis associated with liver disease [16]. In our study, sepsis and a low platelet count were associated with an increased risk of major bleeding. Infection is a frequent complication in critically ill cirrhotic patients, with a cumulative incidence of more than $30 \%$ [17]. Bacterial infection has been associated with an increased risk of early recurrent variceal bleeding [18]. Possible mechanisms to explain this include impaired platelet aggregation associated with sepsis [19] and the release of endogenous heparin-like substances from the endothelium in response to endotoxin [20]. Previous experiments with platelets from cirrhotic patients and from healthy subjects have shown that $50 \times$ $10^{9} / \mathrm{L}$ to $60 \times 10^{9} / \mathrm{L}$ is usually sufficient to preserve adequate thrombin generation, at a level equivalent to the lower limit of the normal range in healthy controls [21]. In our study, the median platelet count was $41.0 \times 10^{9} / \mathrm{L}$ in the group with major bleeding. All of the bleeding events occurred while platelet counts were below $116.0 \times$ $10^{9} / \mathrm{L}$, and platelet counts less than or equal to $37 \times 10^{9} / \mathrm{L}$ on the day of MICU admission were significantly associated with a higher risk of major bleeding (OR, 2.538; $95 \% \mathrm{CI}, 1.084$ to $5.947 ; p=0.028$ ).

The evidence-based clinical practice guidelines for antithrombotic therapy and the prevention of thrombosis published by the American College of Chest Physi- cians recommend using low molecular weight heparin (LMWH) or low-dose unfractionated heparin thromboprophylaxis in critically ill patients. Interestingly, there are no specific recommendations regarding the cirrhotic population [22]. Several studies have suggested that thrombotic complications are common in patients with cirrhosis, and that the use of LMWH is safe and practical $[2,7,9]$. Interestingly, when used for preventing portal vein thrombosis in patients with $\mathrm{LC}$, the anticoagulated group had significantly better outcomes than did the group receiving usual care [23]. Based on the currently available weak evidence, pharmacologic VTE prophylaxis is recommended in cirrhotic patients with a low to intermediate risk of bleeding, but thromboprophylaxis methods should be individualized for patients with a high risk of bleeding $[6,7]$.

Despite evidence supporting VTE prevention, the implementation of VTE prophylaxis appears to be suboptimal. Aldawood et al. [24] reported that $76 \%$ of admitted cirrhotic patients received neither pharmacologic nor mechanical VTE prophylaxis. In our study, only $3.9 \%$ of cirrhosis patients (8/205) received pharmacologic thromboprophylaxis. This low rate may result from a fear of bleeding complications in cirrhotic patients. Bleeding, especially life-threatening hemorrhage such as variceal bleeding, is the most feared complication of anticoagulant therapy, although it has been demonstrated that hemodynamic and mechanical parameters, such as the hepatic vein-portal pressure gradient, varix size, varix appearance (red marks and purple color), together with the severity of the underlying liver disease, are the greatest determinants of the likelihood of such events, not coagulopathy $[9,25]$. 
There are limitations associated with our study. First, we were not able to include unrecorded bleeding events due to the retrospective design of this study. However, we focused on major bleeding events, for which there is only a minimal chance that such clinically significant episodes were not recorded. Second, the pharmacologic thromboprophylaxis rate in the study population was low $(8 / 205,3.9 \%)$, limiting our ability to determine the risks and benefits of VTE prophylaxis in cirrhotic patients. This low utilization of VTE prophylaxis may reflect real-world practice, and more accurate and reliable measures to predict the coagulation status of cirrhotic patients are needed. Third, there was no systematic assessment for VTE, and only $2.9 \%$ of the patients (6/205) underwent testing. The incidence of new VTE in our study was $0.5 \%(1 / 205)$. This is similar to previously reported incidences of $0.5 \%$ to $1.9 \%[2,26]$. Fourth, other coagulation tests such as factor VIII, protein C, or platelet function analysis (PFA-100) were not performed. Unfortunately, these tests are not widely available and are costly to include in the routine screening of critically ill patients with cirrhosis.

Useful risk factors to predict major bleeding in critically ill patients with LC were identified in this study. Commonly used tests have proven to be unreliable for assessing the bleeding risk in cirrhotic patients [16]. Specific testing, such as with a platelet function analyzer (PFA-100) or by thromboelastogram, may be useful in some clinical situations, but are limited by their availability. Among the oldest tests, bleeding time (BT) is prolonged in patients with LC, and has been used to measure primary hemostasis [27]. Boberg et al. [28] showed that prolonged BT was associated with a 5 -fold increase in the risk of hemoglobin drop after liver biopsy. However, correction of the BT did not correlate with decreased hemorrhage, raising questions about the test's reliability [29]. Conventional laboratory tests such as prothrombin time (PT), INR, and activated partial thromboplastin time correlate poorly with bleeding in patients with cirrhosis, whereas PT is normally a well-established indicator of prognosis [16]. Few studies have addressed hemostasis and thrombosis in critically ill cirrhotic patients, and guidelines regarding the management of these patients are lacking. Our findings may shed some light on identifying factors to predict the risk of major bleeding in this patient population and help physicians to make decisions regarding thromboprophylaxis.

In conclusion, major bleeding occurred in $12.2 \%$ of critically ill patients with LC. A low platelet count at MICU admission and sepsis were associated with an increased risk of major bleeding; cirrhotic patients with bleeding had a poor prognosis. Additional well-designed prospective studies are needed to properly understand hemostasis in critically ill cirrhotic patients.

\section{KEY MESSAGE}

1. Major bleeding occurred in about $12 \%$ of critically ill cirrhotic patients admitted to the Medical Intensive Care Unit (MICU).

2. A low platelet count at MICU admission and sepsis were significantly associated with an increased risk of major bleeding during MICU stay.

\section{Conflict of interest}

No potential conflict of interest relevant to this article was reported.

\section{REFERENCES}

1. Yang ZJ, Costa KA, Novelli EM, Smith RE. Venous thromboembolism in cirrhosis. Clin Appl Thromb Hemost 2014;20:169-178.

2. Northup PG, McMahon MM, Ruhl AP, et al. Coagulopathy does not fully protect hospitalized cirrhosis patients from peripheral venous thromboembolism. Am J Gastroenterol 2006;101:1524-1528.

3. Dabbagh O, Oza A, Prakash S, Sunna R, Saettele TM. Coagulopathy does not protect against venous thromboembolism in hospitalized patients with chronic liver disease. Chest 2010;137:1145-1149.

4. Sogaard KK, Horvath-Puho E, Gronbaek H, Jepsen P, Vilstrup H, Sorensen HT. Risk of venous thromboembolism in patients with liver disease: a nationwide population-based case-control study. Am J Gastroenterol 2009;104:96-101.

5. Tripodi A, Mannucci PM. The coagulopathy of chronic 
liver disease. N Engl J Med 2011;365:147-156.

6. Tufano A, Guida A, Di Minno MN, Prisco D, Cerbone AM, Di Minno G. Prevention of venous thromboembolism in medical patients with thrombocytopenia or with platelet dysfunction: a review of the literature. Semin Thromb Hemost 2011;37:267-274.

7. Walsh KA, Lewis DA, Clifford TM, et al. Risk factors for venous thromboembolism in patients with chronic liver disease. Ann Pharmacother 2013;47:333-339.

8. Delgado MG, Seijo S, Yepes I, et al. Efficacy and safety of anticoagulation on patients with cirrhosis and portal vein thrombosis. Clin Gastroenterol Hepatol 2012;10:776-783.

9. Rodriguez-Castro KI, Simioni P, Burra P, Senzolo M. Anticoagulation for the treatment of thrombotic complications in patients with cirrhosis. Liver Int 2012;32:14651476.

10. Roberts LN, Patel RK, Arya R. Haemostasis and thrombosis in liver disease. Br J Haematol 2010;148:507-521.

11. Arnold DM, Donahoe L, Clarke FJ, et al. Bleeding during critical illness: a prospective cohort study using a new measurement tool. Clin Invest Med 2007;30:E93-E102.

12. Arnold DM, Lauzier F, Rabbat C, et al. Adjudication of bleeding outcomes in an international thromboprophylaxis trial in critical illness. Thromb Res 2013;131:204-209.

13. Benvegnu L, Gios M, Boccato S, Alberti A. Natural history of compensated viral cirrhosis: a prospective study on the incidence and hierarchy of major complications. Gut 2004;53:744-749.

14. Gines P, Fernandez J, Durand F, Saliba F. Management of critically-ill cirrhotic patients. J Hepatol 2012;56 Suppl 1:S13-S24.

15. Rudler M, Rousseau G, Benosman H, et al. Peptic ulcer bleeding in patients with or without cirrhosis: different diseases but the same prognosis? Aliment Pharmacol Ther 2012;36:166-172.

16. Caldwell SH, Hoffman M, Lisman T, et al. Coagulation disorders and hemostasis in liver disease: pathophysiology and critical assessment of current management. Hepatology 2006;44:1039-1046.

17. Bernard B, Grange JD, Khac EN, Amiot X, Opolon P, Poynard T. Antibiotic prophylaxis for the prevention of bacterial infections in cirrhotic patients with gastrointestinal bleeding: a meta-analysis. Hepatology 1999;29:1655-1661.

18. Bleichner G, Boulanger R, Squara P, Sollet JP, Parent A. Frequency of infections in cirrhotic patients present- ing with acute gastrointestinal haemorrhage. Br J Surg 1986;73:724-726.

19. Vincent JL, Yagushi A, Pradier O. Platelet function in sepsis. Crit Care Med 2002;30(5 Suppl):S313-S317.

20. Montalto P, Vlachogiannakos J, Cox DJ, Pastacaldi S, Patch $\mathrm{D}$, Burroughs AK. Bacterial infection in cirrhosis impairs coagulation by a heparin effect: a prospective study. J Hepatol 2002;37:463-470.

21. Tripodi A, Primignani M, Chantarangkul V, et al. Thrombin generation in patients with cirrhosis: the role of platelets. Hepatology 2006;44:440-445.

22. Kahn SR, Lim W, Dunn AS, et al. Prevention of VTE in nonsurgical patients: Antithrombotic Therapy and Prevention of Thrombosis, 9th ed: American College of Chest Physicians Evidence-Based Clinical Practice Guidelines. Chest 2012;141(2 Suppl):e195S-e226S.

23. Villa E, Camma C, Marietta M, et al. Enoxaparin prevents portal vein thrombosis and liver decompensation in patients with advanced cirrhosis. Gastroenterology 2012;143:1253-1260.e1-e4.

24. Aldawood A, Arabi Y, Aljumah A, et al. The incidence of venous thromboembolism and practice of deep venous thrombosis prophylaxis in hospitalized cirrhotic patients. Thromb J 2011;9:1.

25. Northup PG, Caldwell SH. Coagulation in liver disease: a guide for the clinician. Clin Gastroenterol Hepatol 2013;11:1064-1074.

26. Gulley D, Teal E, Suvannasankha A, Chalasani N, Liangpunsakul S. Deep vein thrombosis and pulmonary embolism in cirrhosis patients. Dig Dis Sci 2008;53:3012-3017.

27. Violi F, Leo R, Vezza E, Basili S, Cordova C, Balsano F. Bleeding time in patients with cirrhosis: relation with degree of liver failure and clotting abnormalities: C.A.L.C. Group. Coagulation Abnormalities in Cirrhosis Study Group. J Hepatol 1994;20:531-536.

28. Boberg KM, Brosstad F, Egeland T, Egge T, Schrumpf E. Is a prolonged bleeding time associated with an increased risk of hemorrhage after liver biopsy? Thromb Haemost 1999;81:378-381.

29. de Franchis R, Arcidiacono PG, Carpinelli L, et al. Randomized controlled trial of desmopressin plus terlipressin vs. terlipressin alone for the treatment of acute variceal hemorrhage in cirrhotic patients: a multicenter, double-blind study. New Italian Endoscopic Club. Hepatology 1993;18:1102-1107. 